Ann. Abeille, I962, 5 (I), 85-87.

\title{
RAPPORT D'ACTIVITÉ DE LA COMMISSION INTERNATIONALE DE BOTANIQUE APICOLE DE L'U.I.S.B. POUR LES ANNÉES 1959-1961
}

\author{
A. MAURIZIO, T. LOUVE,AUX \\ Station fédérale d'Industrie laitière, Section apicole, Liebefeld-Berne (Suisse). \\ Station de Recherches sur l'Abeille et les Insectes sociaux, Bures-sur-Yvette (Seine-et-Oise).
}

Il y a eu dix ans à l'automne ig62 que fut fonclée à Leamington Spa la Commission internationale de Botanique apicole. Au cours de cette période la commission, après des débuts très modestes, s'est développée en une organisation qui compte aujourd'hui 445 membres appartenant à 30 pays. Flle a tenu, en liaison avec les congrès internationaux d'apiculture, quatre Assemblées générales ordinaires : Copenhagute (r954), Vienne (r956), Bologne (1958) et Madrid (r96r). Les Assemblées générales ont un but principalement administratif, le travail proprement dit se fatsant au sein des groupes de travail.

lin octobre 1959 la commission a perdu l'un de ses membres les plus éminents, le Pr Dr. Ruth BEUTLER qui, depuis la fondation. assurait la direction du groupe de travail "Sécrétion nectarifère."

Pendant la période à laruelle est consacré le présent rapport (1959-196r), ont eu lieu une Assemblée générale ordinaire (Madrid - 22 septembre 196r) et quatre réunions de groupes de travail (Munich - 14/55 juin 1960; Copenhague 12/14 août I960; Vienne 22 aô̂t I960; Madrid 23 septembre 1961). Les publications suivantes ont été adressées aux membres de la Commission.

- Rapport d'activité pour les années i 957 et $195^{8}$ (Zeitschr.f. Bienenforschung, 4 (I) : 209-2 I I, 1959 - Bee World, 40, (2) : 38-4I, 1959 - Ann. Abcille, 2 (2) : I39-144, 1959).

- Circulaire $\mathrm{n}^{0}$ I4 à 17 de la Commission (mars I959- mai I960-février et août 196r).

- Procés-verbal de la réunion du groupe de travail "Protection des Abeilles » à Munich (juin I960).

La $6^{\mathrm{e}}$ liste de publications nouvelles sur l'analyse pollinique et les rapports sur les réunions de Copenhague et de Vienne ( 1960 ) sont en préparation.

Trente-trois membres appartenant à 19 pays ont pris part le 22 septembre 196 i à l'Assemblée générale ordinaire de Madrid. A. Mavrizio et J. Louveaux ont été confirmés dans leurs fonction de Présidente et da Secrétaire de la Commission. L'Union des $\Lambda$ piculteurs suisses acceptant de continuer à couvrir les menues dépenses de la Commission, il reste inutile de percevoir une cotisation et, par voie de conséquence, de désigner un Trésorier.

Trois questions furent sounises à discussion.

- Par suite du décès de R. Beutler, le groupe de travail "Sécrétion nectarifère "se trouve sans direction. R. W. Siruel s'est déclaré disposé à reprendre cette direction. Le Dr SHUEL est élu président du groupe de travail « Sécrétion nectarifère ".

- Deux propositions ont été déposées pour la fondation de nouveaux groupes de travail : 
"Domestication des Bourdons en vue de la pollinisation " et " Étude de la flore mellifère de la zone méditerranéenne sèche ". L'Assemblée charge N. HoLm (1) et H. HaAss, ainsi que P. LAvIE ( ${ }^{2}$ ) et J. Rihar d'essayer de regrouper d'autres collaborateurs et de rendre compte de leurs efforts à la prochaine Assemblée générale.

L'activité des cinq groupes de travail de la Commission de Botanique apicole qui existent actuellement a été la suivante pendant les années 1959-196r.

\section{$\mathrm{I}^{\circ}$ Groupe de Travail pour l'analyse pollinique des miels}

(Rapporteurs : J. Louveaux et A. Maurizio). Dans les dernières années s'est manifesté un ralentissement des travaux sur l'analyse pollinique des miels. Cet état de choses est sans doute en rapport avec le fait que la détermination microscopique de l'origine des micls a atteint en Europe un certain degré de maturité et que de nouveaux développements ne pourront plus être obtenus que par l'introduction de nouvelles méthodes. J. Lovveaux a fait un premier pas dans cette direction par l'introduction d'une méthode combinée (centrifugation et filtration) pour la confection des préparations microscopiques.

Dans le courant de l'année dernière a commencé à paraître, grâce à une collaboration internationale, une série de fiches palynologiques concernant les principales plantes apicoles d'Furope dans la revue "Pollens et Spores" sous la signature de A. Maurizio et J. Louveaux. La première série de Io planches est sortie en I960, la seconde de I4 planches vient de paraître. D'autres séries sont prévues pour 1962 et 1963 ; l'ensemble, qui comprendra une cinquantaine de planches paraîtra en brochure.

S'adaptant aux tendances actuelles, le groupe de travail primitif : "Analyse pollinique " portera à l'avenir le nom de groupe de travail " Recherches sur le miel " et intéressera à côté de l'analyse pollinique, d'autres domaines ayant trait au miel. Un premier symposium sur les questions concernant le miel a eu lieu le 23 septembre 1961 à Madrid en liaison avec la réunion des chercheurs apicoles.

\section{$2^{\circ}$ Groupe de travail pour la section nectarifère}

(Rapporteurs : R. W. Shuel, G. F. Townsend). - Par suite de la maladie, puis du décès de R. BEutLer, les travaux de ce groupe de travail avaient été interrompus. Le nouveau président R. W. SHUEL rendra compte aux membres du groupe de son activité future.

\section{$3^{\circ}$ Groupe de travail pour les recherches sur le miellat}

(Rapporteurs: W. Kloft, F. Ruttner). Faisant suite à la réunion tenue à Fribourg en Brisgau les 4 et 5 juillet 1958 sur les questions concernant les miellats, une seconde réunion a eu lieu à Vienne le 22 août ig60 sur l'initiative de F. Ruttner à l'occasion du XIe Congrès international d'Entomologie. Au cours de ce colloque, présidé par W. KLOFT de Würzbourg, deux communications ont été présentées qui ont traité de nombreuses et intéressantes questions allant de la composition de la sève des tubes criblés, jusqu'à l'importance apicole de miellées forestières. Les rapports présentés parâ̂tront dans le tome 2 des comptes-rendus du XIe Congrès international d'Entomologie, Vienne rg6o. (Les rapports de A. Fossel et F. RutTNer ont été publiés à la suite du congrès dans le numéro spécial du "Bienenvater " consacré à la miellée forestière $-1960 \mathrm{n}^{\circ} 7 / 8$.)

La séance de travail fut complétée par une excursion d'une journée consacrée aux miellats dans la forêt de Vienne, sous la conduite de F. Schremmer (Vienne).

Une nouvelle possibilité de traiter des questions de miellats s'est offerte au IVe Congrès international de l'U. E. I. I. S. à Pavie du 9 au I 4 septembre Ig6r au cours duquel plusieurs communications concernant les rapports entre la production de miellat et les Fournis ont été présentées.

Dans l'ensemble, on a assisté à un effort très satisfaisant pour approfondir et élargir nos connaissances relatives aux problèmes des miellats, ainsi qu'en témoignent les nombreuses et diverses publi-

1) $\mathrm{D}^{r}$ Nörgaard HoLM, Royal Veter. et Agric. College, Experimental Farm, Höjbakkegard pr. Tastrup, Danemark.

2) Dr. P. Lavie, Station expérimentale d'Apiculture, C. R. A. du Sud-Est, Montfavet (Vaucluse), France. 
cations des membres de notre groupe de travail. De plusieurs côtés, on a proposé d'organiser à nouveau la prochaine réunion sur une base interne et non plus dans le cadre d'un grand Congrès. Une telle réunion pourrait avoir lieu éventuellement en 1962 et, au plus tard, en 1963 ; les invitations seront adressées en temps utile. Les suggestions concernant ce projet seront les bienvenues.

\section{$4^{\circ}$ Groupe de travail pour la protection des Abeilles}

(Rapporteurs : K. Stute, J. Evenius). K. Stute a reçu pour mission de la F. A. O. de rédiger un mémorandum sur le thème "Apiculture et traitements phytosanitaires ". En plusieurs chapitres, l'auteur a traité de l'importance de l'apiculture et de la protection des végétaux, de l'action des produits phytosanitaires sur les Abeilles, de l'importance des pertes d'Abeilles dans quelques pays européens, des mesures prises pour la protection des Abeilles et pour la détection des intoxications.

Les 14 et 15 juin 1960 a eu lieu à Munich, en un cercle restreint, une réunion du groupe de travail à laquelle ont assisté les représentants de 7 pays. Les thèmes suivants ont été proposés à la discussion : I) -- Méthodes pour tester les produits quant à leur toxicité pour l'Abeille. 2) Échanges d'informations en ce qui concerne les résultats obtenus jusqu'ici dans les examens de produits antiparasitaires sous le rapport de leur toxicité pour l'Abeille. 3) Classification des produits antiparasitaires sous le rapport de leur toxicité pour l'Abeille. Compte tenu du petit nombre de participants, huit personnes étaient présentes, un très actif échange d'idées a pu avoir lieu et des vues communes ont été mises en évidence sur la plupart des points de l'ordre du jour. Le procès-verbal de cette réunion a été adressé aux membres de la Commission de Botanique apicole.

\section{$5^{\circ}$ Groupe de travail pour les questions de pollinisations}

(Rapporteurs : E. AKerberg, O. HAMmer). Le groupe de travail pour les questions de pollinisation a tenu son premier symposium international en 1960 (I2-I4 août), à Copenhague. Soixante personnes appartenant à $\mathbf{5}$ pays étaient présentes, parmi lesquelles principalement des Scandinaves et autres Européens, ainsi que des Américains (U. S. A. - Canada).

Les rapports ont eu principalement pour thème la pollinisation du Trèfle violet (Trifolium pratense) et de la Luzerne (Medicago sativa), l'osmoguidage et la domestication des Bourdons pour la pollinisation. Cette demière question est aujourd'hui très importante dans beaucoup de pays, car les Bourdons sont mieux adaptés à la pollinisation du Trèfle violet que les Abeilles. Le symposium a été complété par deux excursions qui ont donné aux participants une idée des recherches conduites au Danemark et en Suède sur les questions de pollinisation et d'élevage des Bourdons. Les communications présentées au symposium de Copenhague sont publiées en fin $196 \mathrm{I}$, en un rapport commun. 